\title{
Epilepsy Surgery in Children and Adolescents
}

\section{다 (ㅇ)(ㄱ)(요}

\author{
Authors \\ Georgia Ramantani ${ }^{1,2}$, Josef Zentner ${ }^{3}$

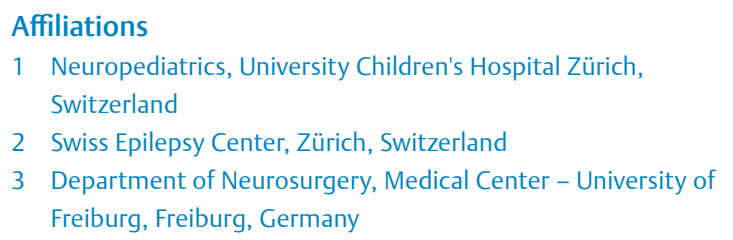

Key words

epilepsy surgery, pharmacoresistance, development, focal cortical dysplasia, glioneuronal tumor

Bibliography

DOI http://dx.doi.org/10.1055/s-0043-102834

Published online: 2017

Neurology International Open 2017; 1: E86-E97

(c) Georg Thieme Verlag KG Stuttgart · New York

ISSN 2511-1795

Correspondence

PD Dr. med. Georgia Ramantani

Neuropädiatrie

Universitäts-Kinderspital

Steinwiesstrasse 75

8032 Zürich

Switzerland

georgia.ramantani@kispi.uzh.ch

\begin{abstract}
Epilepsy surgery has been established in recent years as an effective treatment option for children and adolescents with pharmacoresistant structural epilepsies. Thanks to advances in neurosurgery, anesthesia and intensive care, epilepsy surgery is also possible in infants with excellent results. Epilepsy surgery should be considered in children with structural epilepsies and presurgical evaluation should be initiated at the latest when the criteria for pharmacoresistance are met. Focal cortical dysplasia and glioneuronal tumors are the most common etiologies in pediatric cohorts. Postoperative seizure-freedom depends on completeness of resection. In childhood, multilobular and hemispheric interventions predominate, whereas adults commonly undergo temporal resections. The extent of resections decreases with age. Younger children often require larger resections, but have higher capacities to compensate for neurological deficits, due to functional plasticity. Postoperative seizure freedom depends on the epilepsy syndrome, underlying etiology and accurate demarcation of the epileptogenic zone. Postoperatively, two-thirds of the children remain seizure-free in the long-term. Significant improvements in cognitive development are observed with seizure control. In addition to the development of non-invasive methods for presurgical evaluation, it is crucial to reduce the latency between the establishment of pharmacoresistance, presurgical evaluation and surgical treatment in suitable candidates. Multicentric studies with longer observation intervals are urgently needed in order to identify predictors of seizure freedom and favorable developmental trajectories, to facilitate the selection of optimal candidates and to improve counseling of patients and their families.
\end{abstract}

\section{Introduction}

\section{Epidemiology}

Epilepsy is one of most common neurological disorders in children. The incidence of unprovoked seizures is 44-57 per 100000 population; the incidence of epilepsy is 33-61 per 100000 population. The incidence of epilepsy is considerably higher in the first year of life and remains high in childhood and adolescence to decrease between the third and sixth decade of life. Beyond the first year of life, the incidence of epilepsy remains constant at 46 per 100000 population to decrease markedly after the tenth year of life [1, 2].

\section{Pharmacoresistance}

In a prospective pediatric cohort study with a follow-up of over 10 years, $23 \%$ of children and adolescents diagnosed with epilepsy were drug-resistant at any given time [3]. Although this study indicated a higher proportion of children in remission than previously reported [4], this remission with at least one seizure-free year was achieved on average after 5 or more years, and only after several anticonvulsant trials, whereas two-thirds of these children experienced later recurrences. In this study, only $4 \%$ of patients who did not respond to the second anticonvulsant remained seizurefree over 4 years. Likewise, early remission with anticonvulsants 
followed by subsequent enduring remission at last follow-up (smooth sailing) in a third of the children and adolescents should be taken with caution, since follow-up was limited to a few years.

It is thus presumed that $23 \%$ of children with epilepsy meet the criteria for pharmacoresistance [3], defined as the failure of 2 appropriate treatment trials with well-tolerated and suitably selected anticonvulsants used alone or in combination [5]. Pharmacoresistance is usually established relatively early in the course of epilepsy $[6,7]$, and is often associated with cognitive deficits, psychiatric comorbidities, reduced quality of life and increased SUDEP risk (Sudden Unexpected Death in Epilepsy). Neonatal seizures, high seizure frequency at epilepsy onset, developmental delay and structural lesions correlate with poor chances of remission [8]. In particular, a structural etiology has been identified as the best predictor of pharmacoresistance [9]. Because of this, the initiation of presurgical workup at an early stage is indicated for children with structural epilepsies after the failure of 2 anticonvulsants[10].

\section{Epilepsy surgery}

In recent years, epilepsy surgery has been established as a particularly effective treatment option for children and adolescents with drug-resistant structural epilepsies [11]. Epilepsy surgery should be considered at the latest when pharmacoresistance has been ascertained and presurgical evaluation should be promptly initiated (Cross et al., 2006). The primary goal of epilepsy surgery is the resection or disconnection of the epileptogenic zone, defined as the cortical area that is necessary and sufficient for initiating seizures [12]. In epilepsies associated with a structural lesion, the epileptogenic zone may correspond to the extent of the lesion, but also extend beyond the lesion. In addition to curative surgery, palliative procedures have been developed to avert secondary generalization. Palliative surgery is indicated in cases of diffuse or multifocal seizure origin or if the epileptogenic zone overlaps with eloquent cortical areas $[13,14]$.

Epilepsy onset in early childhood is associated with considerable developmental deficits as well as behavioral issues [15]. Furthermore, longer epilepsy duration is associated with unfavorable developmental outcomes in affected children [16]. In recent years, numerous studies have emphasized the need for early intervention in young children with severe early-onset epilepsy in order to prevent these comorbidities [17-21]. Thanks to the latest technical advances, particularly in neuroimaging, neurosurgery and neuroanesthesia, epilepsy surgery has been established as a safe and effective therapeutic option, including the first years of life [2225]. Despite the so far encouraging results, multicentric studies with longer observation intervals are needed to confirm the positive effects of early epilepsy surgery on children's cognitive development, especially considering the diversity of etiologies associated with a refractory course.

\section{Duration from pharmacoresistance to referral for surgery}

Despite the increased acceptance of epilepsy surgery in the last decades, there is still a substantial delay between the establishment of pharmacoresistance and the referral for presurgical evaluation in an epilepsy center with pediatric expertise [11].The international pediatric epilepsy surgery survey from 2004 showed that only one-third of children underwent surgery within the first 2 years after epilepsy manifestation, whereas two-thirds of these children had presented with seizures in the first 2 years of life [26].In a cohort study in the United States, the incidence of early-onset drugresistant focal epilepsy was 11.3 per 100000 per year, while the incidence of epilepsy surgery in the same cohort was 1.3 per 100000 per year. Only $45 \%$ of children with drug-resistant epilepsy were referred to specialized epilepsy centers for further evaluation [9].

\section{Indications for Epilepsy Surgery}

Although there are generally accepted principles of epilepsy surgery, the precise goals of this intervention are individually defined $[10,27]$. Resective surgery is generally indicated when seizure semiology, EEG and imaging findings allow for a precise delineation of the epileptogenic zone that can be removed without unacceptable neurological and neuropsychological sequelae. The broad range of childhood epileptic syndromes and underlying etiologies as well as the wide variability of clinical and electroencephalographic seizure patterns in the respective age groups define the spectrum of pediatric epilepsy surgery.

In a survey by the ILAE (International League Against Epilepsy) conducted in 2004 [26], focal cortical dysplasias (FCD) (42\%) and glioneuronal tumors (19\%) were the most common etiologies among children and adolescents undergoing surgery in 20 epilepsy centers worldwide. Furthermore, FCD was the most frequent etiology among young children with extratemporal epilepsy. In children and adolescents undergoing temporal lobe resections, FCDs and glioneuronal tumors, i. e. dual pathology, were observed significantly more frequently than isolated hippocampal sclerosis. In addition, this survey provided crucial information regarding the frequency of more rare epilepsy syndromes as well as related etiologies in the pediatric population undergoing epilepsy surgery. Epilepsy surgery was performed in less than $5 \%$ cases in the following rare syndromes: Landau-Kleffner syndrome $(<1 \%)$, ESES (Electrical Status Epilepticus During Slow Sleep) (1\%), Rasmussen encephalitis (3\%), Ohtahara syndrome (<1\%), West syndrome $(4 \%)$, Sturge-Weber syndrome (3\%), and hypothalamic hamartoma (4\%).

- Fig. 1, 2 show the most frequent etiologies of drug-resistant structural epilepsies in infancy and childhood.

\section{Focal cortical dysplasia}

FCDs are the most common cortical malformations in epilepsy surgery and the most frequent etiology in pediatric epilepsy surgery cohorts [26]. FCDs may present as focal, multi-focal or even hemispheric malformations. The FCD-related epileptogenic zone is often more extensive than the MRI-visible lesion [28]. The most common localizations are frontal and temporal, although FCDs can affect all lobes of the brain [29]. The completeness of resection of FCD-related epilepsies determines the postoperative seizure outcome [30]. Despite considerable advances in imaging with development of epilepsy-specific protocols, the accurate demarcation of FCDs remains challenging, especially in the first years of life [17]. Prior to the onset of myelination in the first 6 months of life, there is a crucial time window during which FCDs can be better imaged than in the following 1.5 to 2 years [31]. For this reason, epilepsy-specific imaging should be initiated early in young infants at the first 

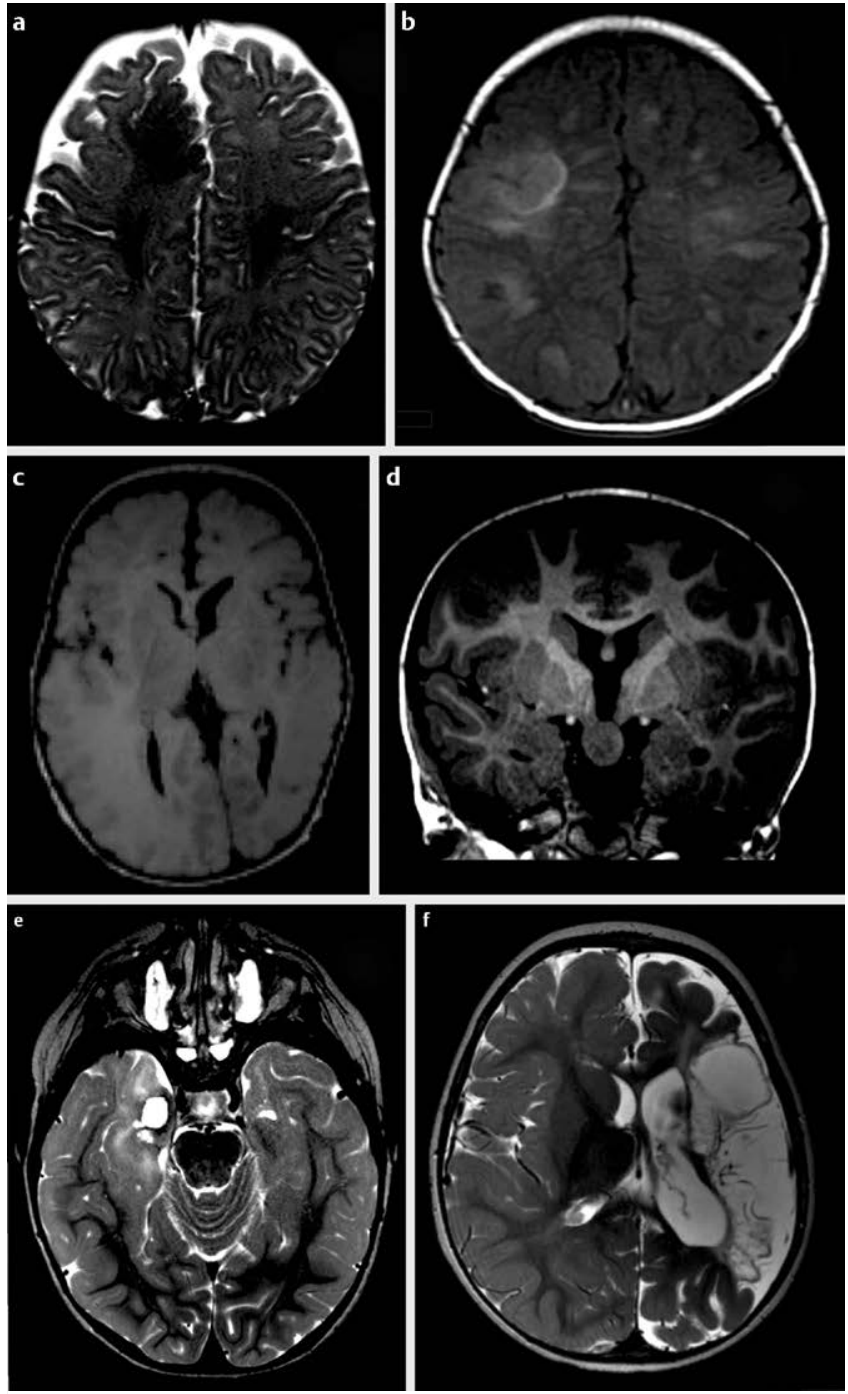

- Fig. 1 The most frequent etiologies of drug-resistant structural epilepsies in childhood. a Right frontal focal cortical dysplasia, b Multiple cortical tubers in tuberous sclerosis, $\mathbf{c}$ Right hemimegalencephaly, d Left hippocampal sclerosis following meningo-encephalitis in infancy, e Right temporal glioneuronal tumor, $\mathbf{f}$ Extensive left frontal cortical scarring following a perinatal cerebrovascular incident.

signs of a severe course. In the case of refractory course and electro-clinical findings suggestive of focal epilepsy, the MRI should be repeated every 6 months, if initially unremarkable, particularly after the age of 24-30 months[32]. FCDs that were not initially detected can become visible with advanced myelination [33]. FCDs constitute a particularly complex group of malformations that vary substantially with respect to anatomo-pathological localization and cellular presentation. In 2011, a new ILAE classification was established to address this diversity of localization and extent of the malformation [34]. FCD Type I is associated with a greater extent across multiple brain lobes as well as with early onset of epilepsy $[34,35]$. FCD Type II is most frequently found in the frontal and parietal lobes, and can manifest as a barely visible bottom-of-sulcus dysplasia or as an extensive lesion comprising several gyri. Similarities have been recently reported at the cellular level between FCD
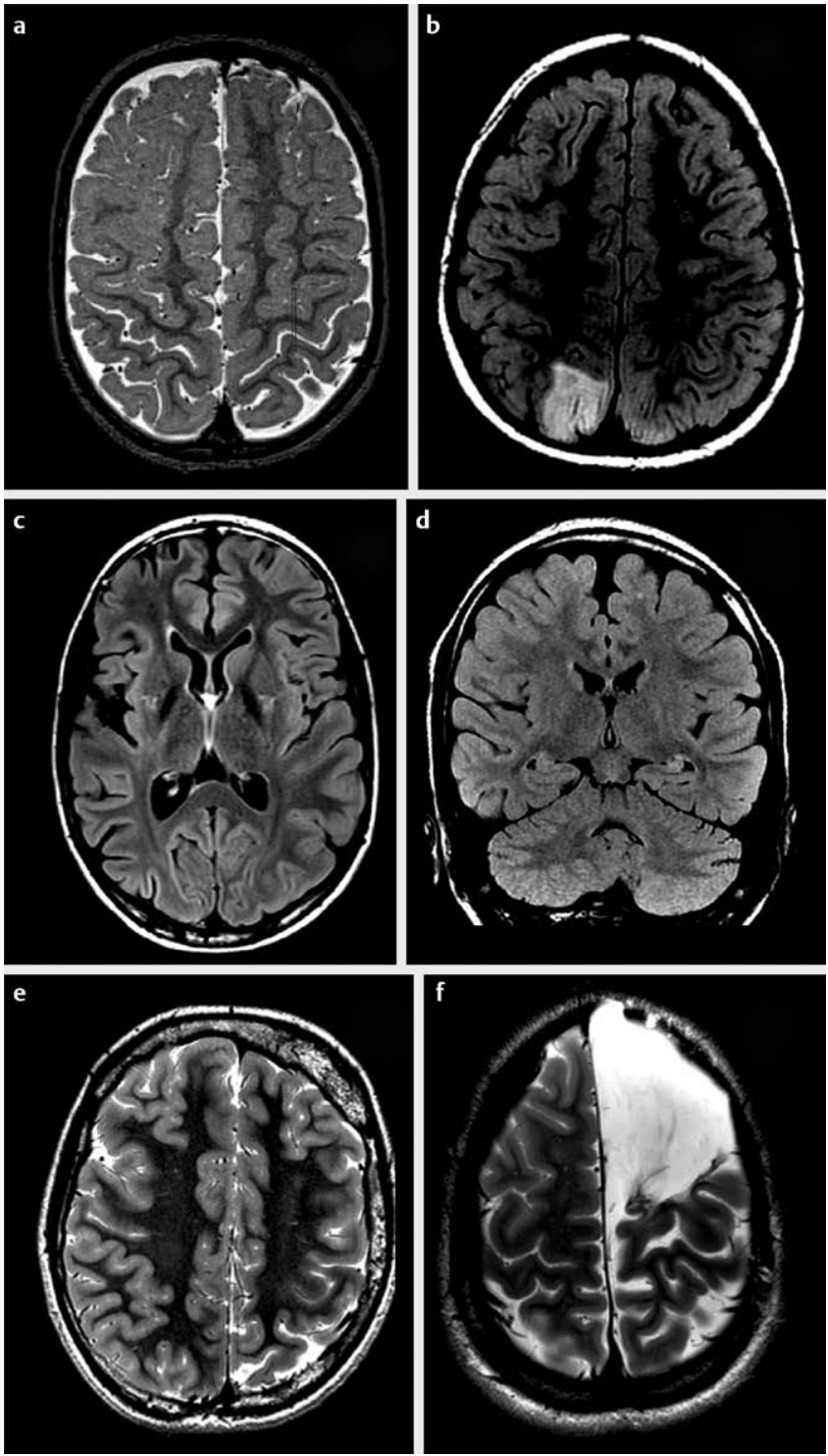

- Fig. 2 The most frequent etiologies of pharmacorefractive structural epilepsies in childhood. a Right frontal cortical dysplasia, b Right parietal DNET, c Right hemispheric Rasmussen encephalitis, d Left hippocampal sclerosis, status after meningeal encephalitis in infancy, e Left parieto-occipital Sturge-Weber syndrome, $\mathbf{f}$ Extended left frontal substance defect after perinatal cerebral hemorrhage.

Type II , hemimegalencephaly and cortical tubers. The new ILAE Type III includes FCDs associated with other lesions, such as hippocampal sclerosis, tumors, vascular, post-traumatic or ischemic lesions.

Despite major diagnostic advances in recent years, FCD-associated epilepsy remains difficult to treat. Optimal outcomes can be achieved by using various diagnostic modalities: $62 \%$ of patients are seizure-free following surgery [28].

\section{Tumors}

Glioneuronal brain tumors (dysembryoplastic neuro-epithelial tumor: DNT, ganglioglioma) are predominantly localized in the temporal lobe and are more frequently associated with refractory 
epilepsy compared to all other pediatric brain tumors [36]. Other less common low-grade gliomas of children and young adults such as supratentorial pilocytic astrocytomas, pleomorphic xanthoastrocytomas, and angiocentric gliomas are also frequently associated with with drug-resistant epilepsy [37]. In addition, FCDs are often associated with these benign pediatric tumors, with a variable prevalence in different studies [36,38]. The completeness of tumor resection determines postoperative seizure freedom $[36,39]$, as long as there is no associated FCD. Epilepsy-specific imaging is needed to rule out any adjacent malformations. In case of discordant seizure semiology or EEG findings, an extraoperative invasive EEG recording or intraoperative electrocorticography (ECoG) should be performed to determine the extent of the resection. Further predictors of postoperative seizure freedom are age at surgery, duration of epilepsy and severity of seizures [40].

Early surgical intervention also plays a significant role in this age group. Longer epilepsy duration correlated with lower cognitive functioning at surgery in a recent pediatric study [36]. This is of great importance, since presurgical functioning determines postsurgical functioning. Cognitive stabilisation or even improvement are expected benefits of successful epilepsy surgery in this context.

\section{Tuberous sclerosis complex}

90 percent of children with tuberous sclerosis present with epilepsy; two-thirds develop pharmacoresistance [41]. Children with tuberous sclerosis and refractory epilepsy are potential candidates for epilepsy surgery. The presence of multiple tubers, multiple seizure types and multifocal epileptiform discharges in scalp EEG represent special challenges in the presurgical evaluation of these patients. As a result, these patients are often excluded from presurgical evaluation, or from surgical intervention. However, epilepsy surgery can achieve seizure freedom in a subgroup of these complex patients, or at least provide some improvement of their condition $[42,43]$. Overall, the results of epilepsy surgery in tuberous sclerosis patients are comparable to those in extratemporal FCDassociated epilepsies. A meta-analysis has demonstrated that $57 \%$ of children are seizure-free after surgery, and an additional $18 \%$ experience a significant improvement in seizure frequency [44]. In these cases, $1-2$ of the multiple tubers were identified as leading tubers and resected. In a recent multi-center study, the extent of resection beyond the tuber correlated with postsurgical seizure freedom [45]. This supports the notion that the epileptogenic zone is not limited to the tuber, but also encompasses adjacent cortical areas. Diagnostic methods that can be particularly useful in the presurgical evaluation of patients with tuberous sclerosis include interictal alpha-11C methyl-L-tryptophan (AMT) PET (Positron Emission Tomography) [46, 47]; ictal SPECT (single photon emission computed tomography) with SISCOM (Subtraction Ictal SPECT co-registered to MRI) [48, 49]; source analysis methods [50]; functional (f)MRI [51], as well as EEG-fMRI (simultaneous EEG and fMRI).

\section{Polymicrogyria}

Polymicrogyria (PMG) is one of the most common cortical malformations, and can be associated with additional malformations such as FCD, hemimegalencephaly, dysgenesis of the corpus callosum, cerebellar hypoplasia, periventricular heterotopia or schizencephaly [52-56]. The underlying etiology is heterogeneous and compri- ses genetic as well as non-genetic fac-tors [57]. PMGs can occur in the vicinity of ischemic lesions, in conjunction with congenital infections (particularly cytomegalovirus infections), metabolic diseases (particularly peroxisomal disorders) as well as gene mutations and copy number variations. PMGs offer a variable clinical presentation depending on the etiology, syndrom-specific characteristics, extent and localization as well as the presence of additional cortical malformations [52, 53, 58, 59]. The age of epilepsy onset varies between the neonatal period and late adulthood, with most patients exhibiting hemiplegia, microcephaly, global developmental delay, and particularly epilepsy, with most patients exhibiting hemiplegia, microcephaly, global developmental delay, and especially epilepsy.

Previous studies regarding seizure freedom after surgery for refractory PMG-associated epilepsy are sparse and their results are somewhat contradictory. This is most likely due to the widely variable epilepsy syndromes, diagnostic methods and surgical procedures considered in each study $[52,53,55,58]$. Postoperative seizure freedom in these studies ranges from 25 to $78 \%$. It remains unclear whether the genetic etiology of certain PMG types [60] can affect postoperative seizure freedom. On the other hand, some PMG types are apparently associated with a favorable long-term outcome despite the occurrence of pharmacoresistance at some point in the course of the disease [61]. In such cases, epilepsy surgery should be considered with caution. Major challenges in surgery for PMG-associated epilepsy are related to (1) the extent of bilateral, multilobar or multifocal malformation, and (2) the presence of epileptogenic as well as electrophysiologically normal cortex within the malformation, with the epileptogenic zone often extending beyond the malformation [53, 62]. Despite this inherent complexity, the outcome of epilepsy surgery is encouraging. Surgical evaluation requires the use of different methods, including invasive EEG recordings [53, 54, 63]. Two recent studies of invasive EEG recordings (SEEG: Stereo-electroence-phalography) in PMGassociated epilepsy underlined the complexity of interrelations between the epileptogenic zone and the PMG $[53,62]$. The epileptogenic zone can encompass the entire PMG or only a part of the PMG as well as distant cortical regions. In some cases, the epileptogenic zone may be limited to remote cortical regions. For this reason, seizure freedom can be achieved with a focal resection even in patients with a multilobar or multifocal PMG, if only part of the PMG is shown to be epileptogenic [64].

\section{Hemispheric syndromes}

Hemispheric syndromes, with refractory epilepsy as a consequence of extensive hemispheric damage are classified as: (1) acquired (e. g. perinatal cerebrovascular incident, hemiconvulsion hemiplegia syndrome, traumatic or infectious causes); (2) congenital (e. g. FCD, PMG, hemimegalencephaly); and (3) progressive (e. g. Rasmussen encephalitis and Sturge-Weber syndrome $[65,66]$. Hemispheric syndromes usually show hemispheric MRI abnormalities, seizure onset in the damaged hemisphere and a contralateral permanent or progressive neurological deficit (motor, language). The integrity of the contralateral hemisphere in hemispherotomy candidates should be verified prior to surgery, since this determines both postsurgical seizure freedom and stabilized or even improved cognitive development [67]. In cases of left-hemispheric pathology, it is important to 
verify language transfer to the contralateral hemisphere by $\mathrm{fMRI}$ or Wada test [65].

\section{MRI-negative epilepsy}

MRI is one of the most important diagnostic tools in the presurgical evaluation [10, 32]. An MRI-visible structural lesion determines the epilepsy classification and sets the indication for presurgical evaluation and, eventually, for epilepsy surgery $[9,68]$.The identification of a structural lesion depends on both the available imaging methods (e. g. high-field MRI, postprocessing) and the experience of the neuroradiologist. The proportion of MR-negative patients with refractory epilepsy that undergo presurgical evaluation varies between $16 \%$ and $32 \%$ [69-74]. A survey by the ILAE Pediatric Epilepsy Surgery Survey Taskforce [26] demonstrated a clear lesion in $77 \%$ of patients, a subtle lesion in $6 \%$, and no lesion in $17 \%$. A meta-analysis revealed a significantly higher proportion of MRInegative cases among children compared to adults (31 vs. $21 \%$ ) [73]. MRI-negative epilepsy is related to poorer chances for a presurgical evaluation and resulting resection [69, 74, 75]. A comprehensive non-invasive presurgical evaluation [76-80] is needed in order to form a valid hypothesis regarding the epileptogenic zone. In the majority of MRI-negative focal epilepsies, invasive EEG recordings are required in order to demarcate the epileptogenic zone $[70,81]$. In subdural EEG recordings, a multifocal or diffuse seizure onset is found 3 times more often in MRI-negative patients compared to MRI-positive cases [82]. Although the likelihood of postoperative seizure freedom is generally reduced among MRInegative patients [73], it still amounts to $40-50 \%$, thus clearly supporting surgical intervention in relation to conservative treatment with continuation of anticonvulsants [83]. Furthermore, some studies of surgery for FCD-associated drug-resistant epilepsy report comparable rates of postsurgical seizure freedom for negative and MR-positive patients [70,71,84].

\section{Presurgical Evaluation}

\section{Scalp EEG recordings}

Based on the guidelines of the ILAE Subcommission for Pediatric Epilepsy Surgery [10], interictal scalp EEG recordings including spontaneous sleep phases are a key component of presurgical evaluation in childhood and adolescence. In addition, simultaneous video-EEG recordings of epileptic seizures are strongly recommended. Particularly in the case of infants and toddlers, sequential EEG recordings are often necessary to establish or rule out a progression. EEG abnormalities in young children with focal, multifocal or bilateral epilepsies are frequently extensive [17], and thus less useful regarding the localization of the epileptogenic zone. Moreover, semiology of focal seizures $n$ the first years of life, is less frequently lateralizing and hardly ever localizing; the level of consciousness is hard to assess [85]. Focal epileptogenic lesions in the first year of life can manifest with infantile spasms in the context of West syndrome $[17,18,21]$.

\section{Invasive EEG recordings}

Invasive recordings are performed to obtain a precise demarcation of the epileptogenic zone in MRI-negative epilepsy as well as in le- sion-related epilepsy or for the functional mapping of adjacent and overlapping eloquent cortical areas [86]. Two essentially different methods of invasive evaluation are available: recordings using subdural strip or grid electrodes $[36,87,88]$ and recordings using intracerebral depth electrodes [36,53]. Placement of grid electrodes requires an open craniotomy, whereas both strip electrodes and depth electrodes can be inserted through burrholes. A combination of subdural and depth electrodes is possible [89]. A recent study reports clinically-relevant complications in $9 \%$ of recordings using subdural electrodes and in $6 \%$ of recordings with depth electrodes. Overall, postoperative complications were reported for $48 \%$ of subdural recordings and $25 \%$ of recordings with depth electrodes [90]. Use of subdural electrodes correlated with an increased risk of postoperative hemorrhage and extraaxial collection. In addition, patients with prior craniotomies demonstrated an increased risk of postoperative hemorrhage. Neurological deficits associated with the invasive recordings were found in $<5 \%$ of patients; less than 1 $\%$ were permanent [90].

Selection of the appropriate method for invasive evaluation depends on individual objectives and limitations. Recordings using depth electrodes enable the assessment of deep structures such as the temporo-mesial areas, the insular cortex or subcortical heterotopias. In addition, depth electrodes are used according to the principles of stereo-encephalography (SEEG) in order to obtain a 3-dimensional illustration of the seizure onset and early spread [91]. On the other hand, recordings with depth electrodes are less suitable for the evaluation of extensive, multilobar malformations or for functional mapping of eloquent cortex. In these cases, subdural recordings are preferable. In the first 3 years of life, recordings with depth electrodes are technically impracticable, since the electrodes cannot be sufficiently fixed to the points of entry due to the particularly thin skull bones $[92,93]$.

Intraoperative electrocorticography (ECoG) [94], is limited to relatively brief recordings in the operating room, thus providing almost exclusively interictal data. Awake surgery, as performed on adults in some epilepsy centers, is unfeasible in pediatric epilepsy surgery due to the lack of cooperation. Moreover, intraoperative recordings are limited to the exposed cortical surface during surgery, whereas deep structures such as the temporal-mesial areas or the insular cortex remain unaccessible.

\section{Imaging}

Due to immature myelination in the first 2 years of life, interpretation of imaging is fraught with difficulty, particularly with respect to the diagnosis of FCDs. Repeated MRI examinations are necessary for this age group in order to identify possible cortical malformations during postnatal brain development.

In addition, functional imaging, such as interictal positron emission tomography (PET), interictal or ictal single-photon emission computed tomography (SPECT), magnetoencephalography (MEG) or functional MRI can provide useful data regarding the localization of the epileptogenic zone. The Wada test can contribute significantly to language lateralization [95].

Finally, source analysis deriving from scalp or invasive EEG recordings as well as MEG recordings can be applied, combined with MRI-based head models for optimal visualization [87, 88, 96, 97]. 


\section{Neuropsychology}

Detailed neuropsychological testing can supplement localization of the epileptogenic zone by identifying deficits while objectifying the functional significance of the affected area, so that the risk of postoperative neuropsychological deficits can be estimated and reduced. This is particularly important, since children with drug-resistant epilepsies who can benefit from epilepsy surgery frequently have accompanying developmental disorders and behavioral problems $[15,17,65,98]$.

\section{Concept of cortical zones}

The epileptogenic zone is the cortical region that is critical for the generation of epileptic seizures [12]. The aim of epilepsy surgery is the complete resection of the epileptogenic zone, sparing the eloquent cortex areas. The complete resection or disconnection of the epileptogenic zone is the necessary and sufficient prerequisite for the achievement of seizure freedom that should be retained even after reduction and discontinuation of anticonvulsive medication. The epileptogenic zone is thus a theoretical concept. The irritative zone is the cortical region generating interictal epileptiform potentials that can be recorded by scalp and intracranial EEG, MEG and EEG-fMRI. The seizure onset zone is the cortical region where epileptic seizures originate. It is determined using scalp and intracranial EEG or ictal SPECT. The symptomatogenic zone is the cortical region that generates the initial ictal symptoms and which can be localized based on an analysis of seizure semiology. An epileptogenic lesion is delineated using high-resolution MRI and may contribute directly to seizure onset due to its intrinsic epileptogenicity, as in the case of FCD, or be secondarily involved by propagation from the surrounding cortical regions. The functional deficit zone is the cortical region that demonstrates functional abnormalities in the interictal period, as indicated by the findings of the neurological and neuropsychological examination.

Resection is generally indicated when (1) seizure semiology, long-term video EEG and imaging findings allow for a clear delineation of the epileptogenic zone, and (2) resection of this area is possible without unacceptable neurological and neuropsychological sequelae. The broad range of childhood epileptic syndromes and related etiologies as well as the wide variability in seizure semiology and electroencephalographic seizure patterns encountered in different age groups account for the main distinctive features of pediatric epilepsy surgery.

\section{Developments in pediatric epilepsy surgery}

Bilateral EEG and MRI findings

Recent developments in the classification of epilepsy syndromes as well as in surgical techniques have expanded the range of epilepsy surgery indications and available procedures [99]. It has been shown in recent years that postsurgical seizure freedom is attainable even in patients with bilateral extensive and diffuse EEG abnormalities and extensive multifocal or multilobar lesions. These observations apply to cases of (1) early-onset catastrophic epilepsy associated with a focal lesion that frequently present with generalized seizure semiology and electroencephalographic abnormalities [17, 36, 87], (2) tuberous sclerosis with multiple tubers, with the resection of the leading tuber resulting in -at least temporary- seizure control [17], and (3) polymicrogyria, with a partial resection guided by invasive EEG recordings resulting in seizure freedom [53].

\section{Extent of resection}

In a single-center study involving 580 cases [100], the outcomes of pediatric epilepsy surgery in Los Angeles in 1986-1997 were compared to those of 1998-2008. In the later time period, the number of curative as well as palliative procedures per year almost doubled, reflecting the dynamic development in pediatric epilepsy surgery. More focal resections were performed in contrast to the dwindling rates of multilobectomies, more patients with tuberous sclerosis were operated, and only rarely did histopathology provide evidence for non-specific gliosis. Moreover, the number of invasive EEGs declined, the outcomes improved and the complication rate decreased. The improvement of seizure outcomes has been ascribed to recent advances in non-invasive presurgical evaluation $[87,88]$, to the improved selection of surgical candidates [65], and particularly to the objective of a complete resection of the epileptogenic lesion [39]. The authors commented that, in the past years, incomplete resection was performed to avoid neurological deficits. For example, temporo-parieto-occipital resections have been performed in children and adolescents with hemispheric epileptogenic lesions and incomplete hemiparesis. In the long-term followup, the majority of patients with incomplete resections of the epileptogenic region suffered recurrent seizures and underwent reoperation relatively soon after the first surgery. In recent years complete resections have gained ground, especially in young children at risk of epileptic encephalopathy, despite the involvement of sensory or motor cortex in some cases [100].

\section{Surgical Interventions}

According to the ILAE [26] survey of epilepsy centers with a pediatric focus in the USA, Europe and Australia, multilobar and hemispheric resections or disconnections dominate in childhood, while temporal resections account for only $25 \%$ of surgeries. This is in stark contrast to adult cohorts with predominantly temporal lobe surgeries and only few extratemporal and hemispheric resections. The extent of the required resection decreases with the age of the surgical candidates.

\section{Focal cortical resection}

The epileptogenic zone and, consequently, the extent of the resection is defined individually depending on the findings of the presurgical evaluation. The complete removal of the epileptogenic zone is the prerequisite for postoperative seizure freedom. In FCD, this corresponds to the complete resection of the epileptogenic lesion. The completeness of FCD resection determines postsurgical seizure freedom [30]. Seizure outcomes following temporal lobe epilepsy surgery are more favorable than the outcomes of extratemporal surgery. Children with MRI-negative epilepsy reportedly have lower chances of seizure freedom. However, the proportion of MRInegative cases has significantly decreased in recent years, due to advances in imaging technologies [11]. In view of the particularly favorable results in children with clearly delineated epileptogenic lesions, e. g. glioneuronal brain tumors, lesionectomy should be undertaken relatively early in the course of epilepsy [36]. 
Temporal lobe epilepsy surgery

Temporal resections account for a quarter of surgical interventions for epilepsy among children and adolescents [26]. Younger age at surgery correlates with higher rates of seizure freedom and more favorable cognitive development [101]. A recent pediatric epilepsy surgery study with follow-up to adulthood reported $85 \%$ seizurefreedom as well as a significant increase of IQ [102]. The improvement in IQ was demonstrated 6 years or longer after epilepsy surgery and was associated with the discontinuation of anticonvulsants.

Glioneuronal brain tumors (40\%) and FCDs (30\%) represent the most frequent etiologies in temporal lobe epilepsy surgery, followed by hippocampal sclerosis (22\%) [26]. In contrast to adult cohorts, isolated hippocampal sclerosis appears in only $7 \%$ of children undergoing surgery. In pediatric cohorts hippocampal sclerosis is diagnosed mainly in adolescents and hardly ever in the first years of life [17]. Dual pathology, i. e. coexistence of hippocampal sclerosis with an additional epileptogenic lesion, is found more frequently in children than adults. In most cases, hippocampal sclerosis is associated with FCDs [28]. Other, less frequent etiologies in temporal lobe epilepsy surgery include polymicrogyria, phacomatosis, atrophic or ischemic lesions [36, 53].

An important issue in temporal lobe epilepsy surgery is the indication of an additional resection of mesial temporal structures in the presence of a clearly delineated epileptogenic lesion, such as a glioneuronal tumor [36]. This issue can often be clarified only with invasive EEG recordings using depth electrodes [53]. This is crucial, since the resection of mesial temporal structures without clear indications of pathology can produce severe cognitive deficits. The results of temporal lobe epilepsy surgery in cases of dual pathology are comparable to those of selective amygdalohippocampectomy, provided that the complete resection of the epileptogenic zone is feasible. The incomplete resection of the epileptogenic zone due to the risk of functional deficits strongly correlates with seizure recurrence in long-term follow-up [39].

\section{Extratemporal epilepsy surgery}

Extratemporal resections account for $20 \%$ of pediatric epilepsy surgery [26]. Despite advances in presurgical evaluation with improved selection of suitable candidates, extratemporal epilepsy surgery is less successful than temporal lobe resections [100]. However, outcomes of extratemporal epilepsy surgery in children and adolescents have considerably improved in recent years and are superior to those in adult cohorts [11]. Cortical malformations represent the leading etiology [28].

Frontal lobe epilepsy surgery: Frontal lobe resections account for $36 \%$ of intralobar epilepsy surgery in children and adolescents [26]. Postoperatively, up to $66 \%$ of patients remain seizure-free, although outcomes can differ according to etiology, localization as well as duration of follow-up. An epileptogenic lesion clearly demarcated in MRI that can be completely resected, due to lack of overlap with eloquent cortical areas, is linked to a particularly favorable prognosis. However, electroencephalographic findings in structural frontal lobe epi-lepsy are often of little help and can lead to the mislateralization and mislocalization of the epileptogenic zone, or even to misclassification of the epileptic syndrome as genetic epilepsy [87].
In a recent study of 158 patients who underwent frontal lobe epilepsy surgery [103] both younger age at surgery (<18 years) and shorter epilepsy duration ( $<5$ years) correlated with higher rates of seizure freedom. In this study, children presented significantly higher rates of seizure freedom compared to adults, especially after early intervention, although FCD was the prevailing etiology in this subgroup. These results can be attributed to the protective effect of early surgery in preventing secondary epileptogenesis. Furthermore, more radical surgery is possible in the first years of life, since neurological deficits can be compensated by increased functional plasticity. This underpins the necessity of early presurgical evaluation and surgical intervention in suitable pediatric candidates.

Posterior cortex epilepsy surgery: Resections in the parietal and/or occipital lobe, sometimes including the posterior part of the temporal lobe, account for $10 \%$ of intralobar epilepsy surgery in children and adolescents [26]. Presurgical evaluation and epilepsy surgery in these brain regions present a significant challenge, due to the rapid connections to other cortical areas and to the functionality of the cortex. Several studies have highlighted the difficulty of delimiting the epileptogenic zone within the parietooccipital and posterior temporal regions, owing to the often nonspecific seizure semiology which, due to rapid propagation, can be mislocalized within the anterior brain regions. In childhood, this challenge is all the greater since electroencephalographic abnormalities are frequently diffuse or generalized and offer little evidence for the localization of the epileptogenic zone.

Postsurgical seizure freedom ranges between $43 \%$ and $86 \%$, depending on the particular cohort and follow-up time, although few studies have been conducted in exclusively pediatric cohorts. In the largest pediatric study of posterior cortex epilepsy surgery to date, including 62 children and adolescents [104], 86\% of patients remained seizure-free in the long-term follow-up of 2-16 years. Postoperative seizure control strongly correlated with a welldelineated, surgically accessible epileptogenic zone. In addition, later epilepsy onset was associated with higher rates of seizure freedom. This may be attributed to the susceptibility of the immature brain to the establishment of extensive epileptic networks, well beyond the epileptogenic lesion. A recent study [105] demonstrated the efficacy of posterior cortex epilepsy surgery: $60 \%$ of the patients remained seizure-free in the long-term follow-up of 1.5-18 years, $30 \%$ were off drugs. Longer epilepsy duration at surgery was identified as the sole independent predictor of seizure recurrence. These results support the early consideration of surgical intervention in children and adolescents with drug-resistant epilepsy originating in the parietal and/or occipital lobes.

\section{Hemispherotomy}

Hemispherotomy, i. e. the functional disconnection of a cerebral hemisphere, currently accounts for $20-40 \%$ of resections in pediatric epilepsy surgery [26]. This procedure is considered in children with hemispheric damage due to a congenital (e. g. FCD, PMG), acquired (e. g. perinatal infarct) or progressive (e. g. Rasmussen encephalitis) etiology, drug-resistant epilepsy and neurological deficits such as hemiparesis and hemianopsia. 
The vast majority of children and adolescents undergoing hemispherotomy remain seizure-free postoperatively [11]. The etiology was identified as the main predictor of seizure freedom [65]. Congenital lesions, especially hemimegalencephaly, are associated with less favorable long-term seizure outcomes. Electroencephalographic or MR-tomographic abnormalities contralateral to the affected hemisphere are generally considered as negative predictors of postsurgical seizure freedom. However, in a recent study it was shown that even contralateral EEG and MRI abnormalities are compatible with postoperative seizure freedom in selected patients [65].

The postsurgical cognitive development of affected children is determined by seizure control, epilepsy duration and presurgical cognitive development. Furthermore, there are indications that postsurgical cognitive functions correlate with the integrity of the contralateral hemisphere.

A recent study [65] identified a subgroup of patients with acquired etiology, older age at surgery and excellent seizure outcomes as "double winners". These results should encourage pediatric neurologists to set aside their reservations regarding possible functional loss and to consider hemispherotomy for older, less affected children or adolescents, especially since these patients might particularly benefit from this surgery.

\section{Special Features of Pediatric Epilepsy Surgery}

\section{Epilepsy surgery in the first years of life}

Epilepsy surgery is an established treatment method for selected pediatric candidates of all age groups with drug-resistant structural epilepsy. However, diagnosis in the first few years of life is fraught with limitations with respect to the MR-tomographic demarcation of epileptogenic lesions. A further challenge in the presurgical evaluation in early childhood are the often diffuse or bilateral interictal epileptiform discharges and electroencephalographic seizure patterns that may arise from focal brain lesions [39,65]. Despite the vast progress in neurosurgery, anaesthesia and intensive care, increased risks are assumed due to the limited blood volume and immature physiology of the developing brain and the extensive procedures often required to achieve seizure freedom in this age group. Nevertheless, there is a trend in offering surgery as soon as intractability is ascertained, including the first years of life

This view is based on the high incidence of epilepsy in the first year of life with one-third of affected children developing pharmacoresistance [6] and on the severity of epilepsy syndromes in this age group [26]. The possibility of epilepsy surgery should be explored at an early stage, considering the cognitive and behavioural impairment associated with early epilepsy onset, longer epilepsy duration, high seizure frequency, continuous epileptiform discharges and polytherapy. Epilepsy surgery during the first years of life benefits from the plasticity of the immature brain. This approach is supported by studies that demonstrate (1) the superiority of surgical treatment compared to pharmacotherapy [19], and (2) the prospects of seizure control and developmental benefits resulting from early intervention [18].
Pediatric epilepsy surgery aims toward seizure control and reduction or discontinuation of anticonvulsants. In view of the devastating effect of seizures and anticonvulsants on the developing brain, a successful surgical intervention is expected to provide longterm benefits regarding cognitive development. After epilepsy surgery, two thirds of children remain seizure-free at long-term follow-up and present a stable [17] or even improved [18] cognitive development. Parents frequently report dramatic improvements in quality of life and an amelioration of social adaptation deficits that go along with seizure control. Morbidity and mortality are low, although extensive resections are often performed. Very young age is not a contraindication for epilepsy surgery.

\section{Reoperations}

The high rate of reoperations is another particular aspect of pediatric epilepsy surgery. This may be partly attributed to the inherent challenges in identifying and delineating FCD, the most prevalent etiology, in MRI, thus increasing the risk of an incomplete first resection. Ten percent of epilepsy surgery in children and adolescents involve reoperations [11]. This is especially relevant in cases of catastrophic epilepsy in the first years of life. After a first epilepsy surgery, $30-40 \%$ of patients continue to suffer seizures; however, only $6-21 \%$ of these children and adolescents undergo reoperation $[17,39]$.

A recent study of reoperations in children and adolescents reported seizure freedom in $61 \%$ of patients, whereas altogether $83 \%$ (Engel I and II: [106]) significantly benefited from reoperation [106]) significantly benefited from reoperation [39]. These outcomes are considerably more favorable compared to those previously reported for adult cohorts with only $20-40 \%$ seizure freedom after reoperation. This is most likely due to the disparities in the underlying pathology and its localization in different age groups. These particularly favorable outcomes of reoperation in childhood derive from the improved MR-tomographic detection of epileptogenic lesions as well as from the latest technological developments. These recent advances have significantly contributed to the accuracy of presurgical evaluation and surgical treatment and expanded the spectrum of potential candidates [28].

Incomplete resections due to fear of functional deficit are often encountered in cases of extensive multilobar or even hemispheric dysplasia, if the epileptogenic zone overlaps with eloquent cortical regions $[17,65]$. The decision for multilobar or hemispheric surgery is further hampered by the insufficient MR-tomographic delimitation of FCD in infants and toddlers, especially in the absence of a neurological deficit. Reoperation in the form of multilobectomy or hemispherotomy offers excellent chances for seizure freedom [39].

Children and adolescents with recurrent seizures after an initial surgery for epilepsy can substantially benefit from reoperation, especially following the incomplete resection of extensive cortical dysplasia. The possibility of reoperation should be promptly considered following seizure recurrence, which in most cases manifests within the first 3 months after epilepsy surgery. Reoperation, when indicated, should be performed at the earliest possible time so that the child can benefit from the higher functional plasticity in early life to compensate for possible neurological deficits. 


\section{Discontinuation of anticonvulsants}

The goals of pediatric epilepsy surgery include seizure freedom, discontinuation of anticonvulsants and improvement of cognitive development [10]. In the first years of life, anti-convulsants can negatively influence brain development by triggering neuronal apoptosis and preventing neurogenesis, synaptogenesis, cell proliferation and migration as well as synaptic plasticity $[107,108]$. In addition, several anticonvulsants have well-known cognitive side effects, particularly affecting attention, vigilance and psychomotor speed $[109,110]$. The cumulative effects of polypharmacotherapy during crucial phases of cognitive development can have a critical influence in affected children and adolescents. The prospect of developmental improvement can encourage anticonvulsant discontinuation in seizure-free patients. A retrospective multicenter study demonstrated that early discontinuation of antiepileptic drugs has no influence on long-term seizure freedom [111]. In some cases, early discontinuation of anticonvulsants can reveal a less favorable outcome. Thus, children and adolescents requiring anticonvulsants in the long-term can be identified, whereas others can be spared unnecessary continuation of drug treatment. A further retrospective multicenter study [112] showed that the initiation of antiepileptic drug withdrawal, the number of drugs reduced, and the complete anticonvulsant withdrawal were associated with improved postoperative cognitive functions.

\section{Summary}

Epilepsy surgery is an effective treatment option for children and adolescents with drug-resistant structural epilepsy. The vast majority of patients benefit substantially from surgical treatment. Very young age, severe developmental delay and psychiatric comorbidities are no contraindications for epilepsy surgery. Likewise, a normal routine MRI should not discourage from pursuing presurgical diagnosis in cases of drug-resistant epilepsy with focal seizures. The extent of the required resection decreases with age. Younger children often require more extensive resections. Postsurgical neurological deficits are generally well-compensated thanks to the increased functional plasticity of the brain. On the other hand, in adolescents with a presurgical neurological deficit, epilepsy surgery can offer excellent chances for seizure freedom and favorable neurocognitive and psychosocial development. In addition to the development of non-invasive methods for presurgical evaluation, it is of utmost importance to shorten the interval between determination of pharmacoresistance, presurgical evaluation, and epilepsy surgery in suitable candidates, in order to improve postsurgical cognitive outcomes. Multicentric studies with longer observation intervals are needed to identify predictors of seizure freedom and improved cognitive development in affected children. This would facilitate the selection of surgical candidates and improve presurgical counseling of patients and their families. Discontinuation of anticonvulsants after successful epilepsy surgery can lead to cognitive improvement without compromising long-term seizure freedom.

\section{Conflicts of Interest}

The authors have no conflicts of interest to disclose.
References

[1] Hauser WA, Annegers JF, Kurland LT. Incidence of epilepsy and unprovoked seizures in Rochester, Minnesota: 1935-1984. Epilepsia 1993; 34: 453-468

[2] Camfield CS, Camfield PR, Gordon K et al. Incidence of epilepsy in childhood and adolescence: a population-based study in Nova Scotia from 1977 to 1985. Epilepsia 1996; 37: 19-23

[3] Berg AT, Rychlik K. The course of childhood-onset epilepsy over the first two decades: a prospective, longitudinal study. Epilepsia 2015; 56: $40-48$

[4] Berg AT, Levy SR, Testa FM et al. Remission of epilepsy after two drug failures in children: a prospective study. Ann Neurol 2009; 65: 510-519

[5] Kwan P, Schachter SC, Brodie M]. Drug-resistant epilepsy. N Engl ] Med 2011; 365: 919-926

[6] Wirrell E, Wong-Kisiel L, Mandrekar J et al. Predictors and course of medically intractable epilepsy in young children presenting before 36 months of age: a retrospective, population-based study. Epilepsia 2012; 53: 1563-1569

[7] Wirrell EC. Predicting pharmacoresistance in pediatric epilepsy. Epilepsia 2013; 54 (Suppl 2): 19-22

[8] Camfield C, Camfield P, Gordon K et al. Outcome of childhood epilepsy: a population-based study with a simple predictive scoring system for those treated with medication. J Pediatr 1993; 122: 861-868

[9] Berg AT, Mathern GW, Bronen RA et al. Frequency, prognosis and surgical treatment of structural abnormalities seen with magnetic resonance imaging in childhood epilepsy. Brain 2009; 132: 27852797

[10] Cross JH, Jayakar P, Nordli D et al. International league against epilepsy, subcommission for paediatric epilepsy surgery, commissions of neurosurgery and paediatrics. Proposed criteria for referral and evaluation of children for epilepsy surgery: recommendations of the subcommission for pediatric epilepsy surgery. Epilepsia 2006; 47: 952-959

[11] Ryvlin P, Cross JH, Rheims S. Epilepsy surgery in children and adults. Lancet Neurol 2014; 13: 1114-1126

[12] Rosenow F, Lüders H. Presurgical evaluation of epilepsy. Brain 2001; 124: $1683-1700$

[13] Cukiert A, Cukiert CM, Burattini JA et al. Long-term outcome after callosotomy or vagus nerve stimulation in consecutive prospective cohorts of children with Lennox-Gastaut or Lennox-like syndrome and non-specific MRI findings. Seizure 2013; 22: 396-400

[14] Benifla M, Otsubo H, Ochi A et al. Multiple subpial transections in pediatric epilepsy: indications and outcomes. Childs Nerv Syst 2006; 22: 992-998

[15] Vasconcellos E, Wyllie E, Sullivan S et al. Mental retardation in pediatric candidates for epilepsy surgery: the role of early seizure onset. Epilepsia 2001; 42: 268-274

[16] Vendrame M, Alexopoulos AV, Boyer K et al. Longer duration of epilepsy and earlier age at epilepsy onset correlate with impaired cognitive development in infancy. Epilepsy Behav 2009; 16: 431-435

[17] Ramantani G, Kadish NE, Strobl K et al. Seizure and cognitive outcomes of epilepsy surgery in infancy and early childhood. Eur ] Paediatr Neurol 2013; 17: 498-506

[18] Loddenkemper T, Holland KD, Stanford LD et al. Developmental outcome after epilepsy surgery in infancy. Pediatrics 2007; 119 : 930-935

[19] Asarnow RF, LoPresti C, Guthrie D et al. Developmental outcomes in children receiving resection surgery for medically intractable infantile spasms. Dev Med Child Neurol 1997; 39: 430-440 
[20] Freitag $\mathrm{H}$, Tuxhorn I. Cognitive function in preschool children after epilepsy surgery: rationale for early intervention. Epilepsia 2005; 46 : 561-567

[21] Jonas R, Asarnow RF, LoPresti C et al. Surgery for symptomatic infant-onset epileptic encephalopathy with and without infantile spasms. Neurology 2005; 64: 746-750

[22] Bulteau C, Otsuki T, Delalande O. Epilepsy surgery for hemispheric syndromes in infants: hemimegalencepahly and hemispheric cortical dysplasia. Brain Dev 2013; 35: 742-747

[23] Otsuki T, Kim H-D, Luan G et al. Surgical versus medical treatment for children with epileptic encephalopathy in infancy and early childhood: Results of an international multicenter cohort study in far-east asia (the FACE study). Brain Dev 2016; 38: 449-460

[24] Kumar RM, Koh S, Knupp K et al. Surgery for infants with catastrophic epilepsy: an analysis of complications and efficacy. Childs Nerv Syst 2015; 31: 1479-1491

[25] Honda R, Kaido T, Sugai K et al. Long-term developmental outcome after early hemispherotomy for hemimegalencephaly in infants with epileptic encephalopathy. Epilepsy Behav 2013; 29: 30-35

[26] Harvey AS, Cross JH, Shinnar S et al. Defining the spectrum of international practice in pediatric epilepsy surgery patients. Epilepsia 2008; 49: 146-155

[27] Wiebe S, Jetté N. Epilepsy surgery utilization: who, when, where, and why? Curr Opin Neurol 2012; 25: 187-193

[28] Guerrini R, Duchowny M, Jayakar P et al. Diagnostic methods and treatment options for focal cortical dysplasia. Epilepsia 2015; 56: 1669-1686

[29] Sisodiya SM. Surgery for malformations of cortical development causing epilepsy. Brain 2000; 123 (Pt 6): 1075-1091

[30] Krsek P, Maton B, Jayakar P et al. Incomplete resection of focal cortical dysplasia is the main predictor of poor postsurgical outcome. Neurology 2009; 72: 217-223

[31] Eltze CM, Chong WK, Bhate $S$ et al. Taylor-type focal cortical dysplasia in infants: some MRI lesions almost disappear with maturation of myelination. Epilepsia 2005; 46: 1988-1992

[32] Gaillard WD, Chiron C, Cross JH et al. Guidelines for imaging infants and children with recent-onset epilepsy. Epilepsia 2009; 50: 2147-2153

[33] Sankar R, Curran JG, Kevill JW et al. Microscopic cortical dysplasia in infantile spasms: evolution of white matter abnormalities. AJNR Am J Neuroradiol 1995; 16: 1265-1272

[34] Blümcke I, Thom M, Aronica E et al. The clinicopathologic spectrum of focal cortical dysplasias: a consensus classification proposed by an ad hoc task force of the ILAE diagnostic methods commission. Epilepsia 2011; 52: 158-174

[35] Hildebrandt M, Pieper T, Winkler P et al. Neuropathological spectrum of cortical dysplasia in children with severe focal epilepsies. Acta Neuropathol (Berl) 2005; 110: 1-11

[36] Ramantani G, Kadish NE, Anastasopoulos C et al. Epilepsy surgery for glioneuronal tumors in childhood: avoid loss of time. Neurosurgery 2014; 74: 648-657 discussion 657

[37] Rudà R, Bello L, Duffau H et al. Seizures in low-grade gliomas: natural history, pathogenesis, and outcome after treatments. Neuro-Oncol 2012; 14 (Suppl 4): iv55-iv64

[38] Nolan MA, Sakuta R, Chuang N et al. Dysembryoplastic neuroepithelial tumors in childhood: long-term outcome and prognostic features. Neurology 2004; 62: 2270-2276

[39] Ramantani G, Strobl K, Stathi A et al. Reoperation for refractory epilepsy in childhood: a second chance for selected patients. Neurosurgery 2013; 73: 695-704
[40] Guerrini R, Rosati A, Giordano F et al. The medical and surgical treatment of tumoral seizures: current and future perspectives. Epilepsia 2013; 54 (Suppl 9): 84-90

[41] Curatolo P, Bombardieri R, Jozwiak S. Tuberous sclerosis. Lancet 2008; 372: 657-668

[42] Lachhwani DK, Pestana E, Gupta A et al. Identification of candidates for epilepsy surgery in patients with tuberous sclerosis. Neurology 2005; 64: 1651-1654

[43] Weiner HL, Carlson C, Ridgway EB et al. Epilepsy surgery in young children with tuberous sclerosis: results of a novel approach. Pediatrics 2006; 117: 1494-1502

[44] Jansen FE, van Huffelen AC, Algra A et al. Epilepsy surgery in tuberous sclerosis: a systematic review. Epilepsia 2007; 48: 1477-1484

[45] Fallah A, Rodgers SD, Weil AG et al. Resective epilepsy surgery for tuberous sclerosis in children: Determining predictors of seizure outcomes in a multicenter retrospective cohort study. Neurosurgery 2015; 77: 517-524 discussion 524

[46] Chugani HT, Luat AF, Kumar A et al. $\alpha$-[11C]-Methyl-L-tryptophanPET in 191 patients with tuberous sclerosis complex. Neurology 2013; 81: 674-680

[47] Rubí S, Costes N, Heckemann RA et al. Positron emission tomography with $\alpha$-[11C]methyl-L-tryptophan in tuberous sclerosis complexrelated epilepsy. Epilepsia 2013; 54: 2143-2150

[48] Koh S, Jayakar P, Resnick T et al. The localizing value of ictal SPECT in children with tuberous sclerosis complex and refractory partial epilepsy. Epileptic Disord 1999; 1: 41-46

[49] Aboian MS, Wong-Kisiel LC, Rank M et al. SISCOM in children with tuberous sclerosis complex-related epilepsy. Pediatr Neurol 2011; 45: $83-88$

[50] Kargiotis O, Lascano AM, Garibotto $V$ et al. Localization of the epileptogenic tuber with electric source imaging in patients with tuberous sclerosis. Epilepsy Res 2014; 108: 267-279

[51] Jacobs ], Rohr A, Moeller F et al. Evaluation of epileptogenic networks in children with tuberous sclerosis complex using EEG-fMRI. Epilepsia 2008; 49: 816-825

[52] Leventer RJ, Jansen A, Pilz DT et al. Clinical and imaging heterogeneity of polymicrogyria: a study of 328 patients. Brain 2010; 133 : 1415-1427

[53] Ramantani G, Koessler L, Colnat-Coulbois S et al. Intracranial evaluation of the epileptogenic zone in regional infrasylvian polymicrogyria. Epilepsia 2013; 54: 296-304

[54] Maillard L, Koessler L, Colnat-Coulbois S et al. Combined SEEG and source localisation study of temporal lobe schizencephaly and polymicrogyria. Clin Neurophysiol Off 2009; 120: 1628-1636

[55] Wang DD, Knox R, Rolston JD et al. Surgical management of medically refractory epilepsy in patients with polymicrogyria. Epilepsia 2016; 57: 151-161

[56] Wieck G, Leventer RJ, Squier WM et al. Periventricular nodular heterotopia with overlying polymicrogyria. Brain 2005; 128 : 2811-2821

[57] Guerrini R, Barba C. Malformations of cortical development and aberrant cortical networks: epileptogenesis and functional organization. J Clin Neurophysiol Soc 2010; 27: 372-379

[58] Shain C, Ramgopal S, Fallil Z et al. Polymicrogyria-associated epilepsy: a multicenter phenotypic study from the epilepsy phenome/genome project. Epilepsia 2013; 54: 1368-1375

[59] Castaño de la Mota C, Rojas MLR-F, Peñas JJG et al. Polymicrogyria: epidemiology, neurological and anatomical factors and clinical outcome in a series of 34 cases. An Pediatría Barc Spain 2003 2011; 75: $358-364$ 
[60] Stutterd CA, Leventer R]. Polymicrogyria: a common and heterogeneous malformation of cortical development. Am J Med Genet C Semin Med Genet 2014; 166C: 227-239

[61] Guerrini R, Genton P, Bureau M et al. Multilobar polymicrogyria, intractable drop attack seizures, and sleep-related electrical status epilepticus. Neurology 1998; 51: 504-512

[62] Chassoux F, Landre E, Rodrigo $S$ et al. Intralesional recordings and epileptogenic zone in focal polymicrogyria. Epilepsia 2008; 49: 51-64

[63] Rikir E, Koessler L, Gavaret M et al. Electrical source imaging in cortical malformation-related epilepsy: a prospective EEG-SEEG concordance study. Epilepsia 2014; 55: 918-932

[64] Cossu M, Pelliccia V, Gozzo F et al. Surgical treatment of polymicrogyria-related epilepsy. Epilepsia 2016; 57: 2001-2010

[65] Ramantani G, Kadish NE, Brandt A et al. Seizure control and developmental trajectories after hemispherotomy for refractory epilepsy in childhood and adolescence. Epilepsia 2013; 54: 10461055

[66] Moosa ANV, Gupta A, Jehi L et al. Longitudinal seizure outcome and prognostic predictors after hemispherectomy in 170 children. Neurology 2013; 80: 253-260

[67] Boshuisen K, van Schooneveld MM], Leijten FSS et al. Contralateral MRI abnormalities affect seizure and cognitive outcome after hemispherectomy. Neurology 2010; 75: 1623-1630

[68] Bast T. Outcome after epilepsy surgery in children with MRI-negative non-idiopathic focal epilepsies. Epileptic Disord 2013; 15: 105-113

[69] Bien CG, Szinay M, Wagner ] et al. Characteristics and surgical outcomes of patients with refractory magnetic resonance imagingnegative epilepsies. Arch Neurol 2009; 66: 1491-1499

[70] McGonigal A, Bartolomei F, Régis ] et al. Stereoelectroencephalography in presurgical assessment of MRI-negative epilepsy. Brain 2007; 130: 3169-3183

[71] Paolicchi JM, Jayakar P, Dean P et al. Predictors of outcome in pediatric epilepsy surgery. Neurology 2000; 54: 642-647

[72] Siegel AM, Jobst BC, Thadani VM et al. Medically intractable, localization-related epilepsy with normal MRI: presurgical evaluation and surgical outcome in 43 patients. Epilepsia 2001; 42: 883-888

[73] Téllez-Zenteno JF, Hernández Ronquillo L, Moien-Afshari F et al. Surgical outcomes in lesional and non-lesional epilepsy: A systematic review and meta-analysis. Epilepsy Res 2010; 89: 310-318

[74] Teutonico F, Mai R, Veggiotti P et al. Epilepsy surgery in children: evaluation of seizure outcome and predictive elements. Epilepsia 2013; 54 (Suppl 7): 70-76

[75] Englot D], Breshears JD, Sun PP et al. Seizure outcomes after resective surgery for extra-temporal lobe epilepsy in pediatric patients. J Neurosurg Pediatr 2013; 12: 126-133

[76] Focke NK, Bonelli SB, Yogarajah M et al. Automated normalized FLAIR imaging in MRI-negative patients with refractory focal epilepsy. Epilepsia 2009; 50: 1484-1490

[77] Kim H, Kankirawatana P, Killen J et al. Magnetic source imaging (MSI) in children with neocortical epilepsy: surgical outcome association with 3D post-resection analysis. Epilepsy Res 2013; 106: 164-172

[78] Thivard L, Bouilleret V, Chassoux F et al. Diffusion tensor imaging can localize the epileptogenic zone in nonlesional extra-temporal refractory epilepsies when [(18)F]FDG-PET is not contributive. Epilepsy Res 2011; 97: 170-182

[79] Chassoux F, Rodrigo S, Semah F et al. FDG-PET improves surgical outcome in negative MRI Taylor-type focal cortical dysplasias. Neurology 2010; 75: 2168-2175

[80] Nguyen DK, Rochette E, Leroux J-M et al. Value of 3.0T MR imaging in refractory partial epilepsy and negative 1.5T MRI. Seizure 2010; 19: 475-478
[81] Alarcón G, Valentín A, Watt C et al. Is it worth pursuing surgery for epilepsy in patients with normal neuroimaging? J Neurol Neurosurg Psychiatry 2006; 77: 474-480

[82] Kalamangalam GP, Pestana Knight EM, Visweswaran S et al. Noninvasive predictors of subdural grid seizure localization in children with nonlesional focal epilepsy. J Clin Neurophysiol Off Publ Am Electroencephalogr Soc 2013; 30: 45-50

[83] Jayakar P, Dunoyer C, Dean P et al. Epilepsy surgery in patients with normal or nonfocal MRI scans: integrative strategies offer long-term seizure relief. Epilepsia 2008; 49: 758-764

[84] Lazow SP, Thadani VM, Gilbert KL et al. Outcome of frontal lobe epilepsy surgery. Epilepsia 2012; 53: 1746-1755

[85] Nordli DR. Varying seizure semiology according to age. Handb Clin Neurol 2013; 111: 455-460

[86] Ramantani G, Maillard L, Koessler L. Correlation of invasive EEG and scalp EEG. Seizure 2016; 41: 196-200

[87] Ramantani G, Dümpelmann M, Koessler L et al. Simultaneous subdural and scalp EEG correlates of frontal lobe epileptic sources. Epilepsia 2014; 55: 278-288

[88] Ramantani G, Cosandier-Rimélé D, Schulze-Bonhage A et al. Source reconstruction based on subdural EEG recordings adds to the presurgical evaluation in refractory frontal lobe epilepsy. Clin Neurophysiol 2013; 124: 481-491

[89] Munyon CN, Koubeissi MZ, Syed TU et al. Accuracy of frame-based stereotactic depth electrode implantation during craniotomy for subdural grid placement. Stereotact Funct Neurosurg 2013; 91 : 399-403

[90] Schmidt RF, Wu C, Lang M] et al. Complications of subdural and depth electrodes in 269 patients undergoing 317 procedures for invasive monitoring in epilepsy. Epilepsia 2016; 57: 1697-1708

[91] Bancaud J, Talairach J. Methodology of stereo EEG exploration and surgical intervention in epilepsy. Rev Otoneuroophtalmol 1973; 45: $315-328$

[92] Cossu M, Schiariti M, Francione S et al. Stereoelectroencephalography in the presurgical evaluation of focal epilepsy in infancy and early childhood. J Neurosurg Pediatr 2012; 9: 290-300

[93] Taussig D, Dorfmüller G, Fohlen M et al. Invasive explorations in children younger than 3 years. Seizure 2012; 21: 631-638

[94] Penfield WG, Jasper HH. Epilepsy and the functional anatomy of the human brain. Boston, Mass, USA: Little Brown \& Co; 1954

[95] Wagner K, Hader C, Metternich B et al. Who needs a wada test? Present clinical indications for amobarbital procedures. J Neurol Neurosurg Psychiatry 2012; 83: 503-509

[96] Bast T, Ramantani G, Boppel T et al. Source analysis of interictal spikes in polymicrogyria: loss of relevant cortical fissures requires simultaneous EEG to avoid MEG misinterpretation. Neurolmage 2005; 25 : $1232-1241$

[97] Ramantani G, Boor R, Paetau R et al. MEG versus EEG: influence of background activity on interictal spike detection. J Clin Neurophysiol 2006; 23: 498-508

[98] Besag F, Gobbi G, Aldenkamp A et al. Psychiatric and behavioural disorders in children with epilepsy (ILAE task force report): Subtle behavioural and cognitive manifestations of epilepsy in children. Epileptic Disord 2016: dio 10.1684/epd.2016.0816

[99] Cukiert A, Rydenhag B, Harkness W et al. Technical aspects of pediatric epilepsy surgery: Report of a multicenter, multinational web-based survey by the ILAE task force on pediatric epilepsy surgery. Epilepsia 2016; 57: 194-200

[100] Hemb M, Velasco TR, Parnes MS et al. Improved outcomes in pediatric epilepsy surgery: the UCLA experience, 1986-2008. Neurology 2010; 74: $1768-1775$ 
[101] Gleissner U, Sassen R, Schramm J et al. Greater functional recovery after temporal lobe epilepsy surgery in children. Brain 2005; 128 : 2822-2829

[102] Skirrow C, Cross JH, Cormack F et al. Long-term intellectual outcome after temporal lobe surgery in childhood. Neurology 2011; 76 : 1330-1337

[103] Simasathien T, Vadera S, Najm I et al. Improved outcomes with earlier surgery for intractable frontal lobe epilepsy. Ann Neurol 2013; 73: 646-654

[104] Liava A, Mai R, Tassi L et al. Paediatric epilepsy surgery in the posterior cortex: a study of 62 cases. Epileptic Disord Int Epilepsy J Videotape 2014; 16: 141-164

[105] Ramantani G, Stathi A, Brandt A et al. Posterior cortex epilepsy surgery in childhood and adolescence: Predictors of long-term seizure outcome. Epilepsia 2017; 58: 412-419

[106] Engel ] Jr, Van Ness PC, Rasmussen TB et al. Outcome with respect to epileptic seizures. In: Engel J Jr (ed.). Surgical treatment of the epilepsies. New York: Raven Press; 1993: 609-621
[107] Ikonomidou C, Turski L. Antiepileptic drugs and brain development. Epilepsy Res 2010; 88: 11-22

[108] Kaindl AM, Asimiadou S, Manthey D et al. Antiepileptic drugs and the developing brain. Cell Mol Life Sci CMLS 2006; 63: 399-413

[109] Loring DW, Meador KJ. Cognitive side effects of antiepileptic drugs in children. Neurology 2004; 62: 872-877

[110] Meador KJ. Cognitive outcomes and predictive factors in epilepsy. Neurology 2002; 58: S21-S26

[111] Boshuisen K, Arzimanoglou A, Cross JH et al. Timing of antiepileptic drug withdrawal and long-term seizure outcome after paediatric epilepsy surgery (TimeToStop): a retrospective observational study. Lancet Neurol 2012; 11: 784-791

[112] Boshuisen K, van Schooneveld MM], Uiterwaal CSPM et al. Intelligence quotient improves after antiepileptic drug withdrawal following pediatric epilepsy surgery. Ann Neurol 2015; 78: 104-114 